$-75-$

\section{ASSESSMENT OF TRICUSPID REGURGITATION BY REAL-TIME TWO DIMENSIONAL DOPPLER FLOW IMAGING TECHNIQUE AND ITS CLINICAL APPLICATION}

Kazuya Yamamoto, Kunio Miyatake, Shiro Izumi, Toshihiro Misawa, Mitsushige Ohta, Kohei Kawazoe, Tsuyoshi Fujita, Shintaro Beppu, YungDae Park, Seiki Nagata, Naokazu Kinoshita, Hiroshi Sakakibara, Yasuharu Nimura.

National Cardiovascular Center, Osaka.

Real-time two dimensional Doppler flow imaging technique (2DDF) was applied to the assessment of severity of tricuspid regurgitation (TR). study group comprised 109 cardiac patients. Severity of TR was classified into 4 steps on the basis of the maximum distance (D) and flow area (A) of regurgitant flow image (On distance, $1+: D<2.0 \mathrm{~cm}, 2+: 2.0 \mathrm{~cm} \leq \mathrm{D}<4.0 \mathrm{~cm}, 3+: 4.0 \mathrm{~cm} \leq \mathrm{D}<6.0 \mathrm{~cm}$, $4+: D \geqq 6,0 \mathrm{~cm}$, and on area, $1+: A<2.0 \mathrm{~cm}^{2}, 2+; 2.0 \mathrm{~cm}^{2} \leq \mathrm{A}$ $\left.<4.0 \mathrm{~cm}^{2}, 3+: 4.0 \mathrm{~cm}^{2} \leqq A<8.0 \mathrm{~cm}^{2}, 4+: A \geqq 8.0 \mathrm{~cm}^{2}\right)$.

1) In 47 cases, severity of TR was classified into 4 steps by right ventriculography (RVG) according to the modified criteria of sellers classification for mitral regurgitation. significant correlation was found between the severity of TR by $2 \mathrm{DDF}$ and by RVG

(D: $r=0.84, A: r=0.89$ ).

2) Fifty cases with mitral and/or aortic valvular disease combined with functional TR underwent open heart surgery. Preoperative 2DDF study demonstrated $3+$ (moderately severe) to $4+$ (severe) $\mathrm{TR}$ in 19 and $2+$ (moderate) to $1+$ (mild) $\mathrm{TR}$ in 31 cases. Fifteen of 19 cases (79\%) with preoperative $3+$ to $4+T R$ and 2 of 31 cases $(6 \%)$ with $1+$ to $2+T R$ had tricuspid annuloplasty (TAP) by intraoperative judgment of surgeon. Thus, patients with TR of $3+$ or more were considered to be candidate for TAP. Among 17
cases who had TAP, postoperative $2 \mathrm{DDF}$ showed decrease in the grade of TR in 2 cases by 3 to 4 steps, in 4 cases by 2 steps and in 4 cases by one step. In contrast no significant change in the grade of TR was noted in 33 cases who underwent left heart surgery but without TAP cone step increase in 8 cases, one step decrease in 10 cases and no change in 15 cases).

In conclusion, 2DDF is available for noninvasive assessment of severity of $T R$ and prediction of necessity of TAP in cases of functional TR.

$-76-$

CLINICAL SIGNIFICANCE OF HEPATIC VEIN FLOW IN RIGHT HEART VOLUME-OVERLOAD DISORDERS

HIROSAWA

KICHIRO SAKAI, KENJI NAKAMURA, KOSHICHIRO

Department of Cardiology, The Heart Inst. of Japan, Tokyo Women's Medical College

8-1 Kawada-cho, Shinjuku-ku, Tokyo 162

To evaluate hemodynamics of right heart volume-overload disorders (RHVO)-tricuspid regurgitation(TR) and $A S D$, we analyzed hepatic vein flow(HVF) by pulsed Doppler echocardiography. In control subjects, HVF had a toward flow(A) by atrial kick followed by biphasic away flow with systolic flow(S) greater than diastolic flow(D). In 89 patients(pts) with TR which was graded as mild(53), moderate(21) and severe(15) by RV-graphy, several abnormal HVF patterns were observed. S became smaller and got reversed as the severity of TR became worse. Thirty one of the $36 \mathrm{pts}(86 \%)$ with moderate or severe TR showed reversed S. Also, Doppler shifts(DS) correlated well with right atrial pressure(RAP) and RV end-diastolic pressure (RVEDP) in moderate or severe TR groups ( $r=0.80$, $0.72 ; \mathrm{p} 0.001$, respectively). In 31 pts with ASD, $A, S, D$ and late systolic toward flow(S') were significantly greater $(\mathrm{p} 0.001)$ than in control group. But there were no good correlations between DS and shunt ratio or pressure. In conclusion, two completely different HVF types in RHVO were observed. In cases with TR, HVF pattern enabled us to assess the severity of $\mathrm{TR}$, and to estimate RAP and RVEDP.

\section{Chairman}

$\begin{array}{ll}77-82 & \text { Y. Kaneko, Yokohama } \\ 83-88 & \text { Y. Masuyama, Wakayama }\end{array}$

\section{$-77-$ \\ THE EFFECT OF CALCIUM (Ca) INTAKE ON SODIUM RETENTION IN DOCA-SALT HYPER- TENSIVE RATS}

Takehiro Omatsu, Hiroshi Sano, Hiroshi Suzuki, Takaaki Motoyama, Keizo Kawaguchi, Komei Saito*, Junji Yamanishi, Yoshitaka Nishimura, Hsieh Shao-Ming, Yutaka Furuta, Hisashi Fukuzaki

The First Department of Internal Medicine, Kobe University School of Medicine, Kobe. *Internal Medicine, Hidaka Hospital, Hyogo.

Epidemiologic and animal studies have suggested the hypotensive effect of high Ca intake. We measured the effect of high $\mathrm{Ca}$ diet on blood pressure (BP), cardiac rate (CR), urine volume (UV) and 24 hours urinary sodium excretion (U-Na) in 6 weekold DOCA-salt hypertensive rats (DSR; $n=55$ ) and age matched controls $(n=23)$. DSR were fed with or without added $\mathrm{CaCl}_{2}$ for 4 weeks and at the end of the study, measurements of extracellular fluid volume (ECFV), total body sodium ( $\mathrm{T}-\mathrm{Na}$ ), red cell sodium ( $\mathrm{R}-\mathrm{Na}$ ) and hormonal parameters were performed. High $\mathrm{Ca}$ intake resulted in a significant suppression in BP $(p<0.001)$ and an increase in UV and U-Na $(p<0.0220 .001)$ in DSR. ECFV was reduced from $33.0 \pm 0.6 \mathrm{ml} / 100 \mathrm{~g}$ body weight to $31.0 \pm 0.5$ ( $p<$ $0.05)$. T-Na and R-Na were also decreased ( $p<0.005$ and $\mathrm{p}<0.05$, respectively) by $\mathrm{Ca}$ supplement. The decrease in catecholamine contents of hearts (heart-CA) and AVP contents of hypophysis in DSR were improved, while $\mathrm{CR}$ was decreased. $\mathrm{R}-\mathrm{Na}$ correlated positively to $B P(r=0.675, p<0.001)$ and inversely to heart-CA. Survival rate in Ca supplemented DSR was higher than that in DSR. These results provide an evidence that high $\mathrm{Ca}$ intake may attenuate the BP increase inDSR by inducing natriuresis and normalization of enhanced activity of sympathetic nerve system, and also suggest that these effects of Ca supplement may, at least in part, contribute to the improvement of mortality in DSR.

$-78-$
THE EFFECT OF RAT ATRIOPEPTIN III ON
RAT GLOMERULAR ANGIOTENSIN II RE-
CEPTOR PROPERTIES AND PLASMA
ANGIOTENSIN II CONCENTRATION IN

Japanese Circulation Journal Vol. so, June 1986 